\title{
Teor, rendimento e composição química do óleo essencial de plantas de manjericão submetidas ao estresse salino com $\mathrm{NaCl}$
}

\author{
ALVES, L.S."*; PAZ, V.P.S.'; SILVA, A.J.P.;; OLIVEIRA, G.X.S.'; OLIVEIRA, F.E.R.'; AMORIM, E.L.1 \\ 1Universidade Federal do Recôncavo da Bahia, Rua Rui Barbosa, 710 - Centro, Cruz das Almas - BA, 44380- \\ 000. Autor para correspondência: lusuzart85@yahoo.com.br
}

\begin{abstract}
RESUMO: O objetivo do presente trabalho foi avaliar o teor, o rendimento e a composição química do óleo essencial de manjericão (Ocimum basilicum L.) submetido ao estresse salino por $\mathrm{NaCl}$. Para tanto, o experimento foi conduzido em casa de vegetação, sob delineamento inteiramente casualizado, caracterizado por 5 (cinco) tratamentos de $\mathrm{NaCl}$, adicionados à água de irrigação (0,01 - controle,T1; 1,2 - T2; 2,3 - T3; 3,4 - T4 e 4,5 dS m-1- T5, correspondendo respectivamente a 0,$1 ; 12,0 ; 23,0 ; 34,0$ e $45,0 \mathrm{mM}$ ), com 4 (quatro) repetições. Aos 55 dias após o transplantio, as plantas foram colhidas, colocadas em sacos de papel e levadas a estufa de secagem com fluxo de ar forçado a $45^{\circ} \mathrm{C}$, por 10 dias até atingirem massas constantes. Após a secagem, foi realizada a pesagem da biomassa seca da parte aérea, misturada, moída em triturador elétrico de facas e posteriormente realizada a extração do óleo essencial para análise do teor, rendimento e composição química. O aumento do estresse por $\mathrm{NaCl}$ não alterou o teor e a composição química do óleo essencial, cujo rendimento reduziu $12,93 \%$ a cada acréscimo unitário dos níveis de salinidade. O cinamato de metila, cujo teor médio encontrado foi de $55 \%$, e o metil chavicol, com $12 \%$, foram os principais compostos identificados nas plantas de manjericão.
\end{abstract}

Palavras-chave: salinidade, Ocimum basilicum L., metabolismo secundário

\begin{abstract}
Content, yield and chemical composition of essential oil of sweet basil plants subjected to $\mathrm{NaCl}$ saline stress. This study aimed to evaluate the content, yield and chemical composition of the essential oil of sweet basil (Ocimum basilicum L.) plants subjected to $\mathrm{NaCl}$ saline stress. The experiment was carried out in a greenhouse, in a completely randomized design, with 5 (five) $\mathrm{NaCl}$ treatments added to the irrigation water (0.01 - control, T1; 1.2 - T2; 2.3 - T3; 3.4 - T4 and $4.5 \mathrm{dS} \mathrm{m}^{-1}$ - T5, corresponding respectively to $0.1 ; 12.0 ; 23.0 ; 34.0$ and $45.0 \mathrm{mM}$ ) and 4 (four) replicates. At 55 days after transplanting, plants were harvested, put in paper bags and taken to a forced-air oven at $45^{\circ} \mathrm{C}$ for 10 days, until reaching constant mass. After drying, the dry biomass of the aerial part was weighed, mixed, grinded in an electric blade grinder and later taken to an essential oil extraction so that its content, yield and chemical composition could be analyzed. The increase of $\mathrm{NaCl}$ stress did not change neither the content nor the chemical composition of the essential oil, whose yield was reduced by $12.93 \%$ for each unit increase in the salinity level. The methyl cinnamate, with an average content of $55 \%$, and the methyl chavicol, with $12 \%$, were the main compounds identified in the sweet basil plants.
\end{abstract}

Keywords: salinity, Ocimum basilicum L., secondary metabolism

\section{INTRODUÇÃO}

A região semiárida do Nordeste Brasileiro caracteriza-se por apresentar insuficiência hídrica, bem como disponibilidade de água com teor elevado de sais, além de temperaturas elevadas, esses são fatores limitantes ao desenvolvimento de inúmeras espécies. A irrigação com água salina leva a uma alta concentração de sais no solo, além de reduzir o potencial hídrico, podendo provocar efeitos tóxicos nas plantas, causando distúrbios funcionais e injurias no metabolismo (Silva et al., 2003). De acordo com Rhoades et al. (2000), a utilização de espécies tolerantes a salinidade e a adoção de práticas de manejo de cultivo, do solo e da água tem propiciado o uso de águas salinas na irrigação de diversas culturas.

Diante de um contexto de escassez de 
recursos hídricos de qualidade, bem como a importância das plantas medicinais, com destaque para o cultivo do manjericão, devido a importância do seu óleo essencial para as indústrias, optouse pelo estudo dessa espécie em condições de estresse salino.

O manjericão (Ocimum basilicum L.) pertence à família Lamiaceae, caracteriza-se como subarbusto aromático, anual, ereto, ramificado, de $30-50 \mathrm{~cm}$ de altura, nativo da Ásia tropical e introduzido no Brasil pela colônia italiana. Cultivado em quase todo o Brasil em hortas domésticas para uso condimentar e medicinal, sendo comercializado na forma fresca em feiras e supermercados (Lorenzi \& Matos, 2008).

Entre as ervas aromáticas, o manjericão produz seu óleo essencial ou princípio ativo através do metabolismo secundário, que é ativado nas plantas como reação a algum tipo de estresse (Lawrence, 1992; Garlet, 2007; Azevedo \& Moura, 2010). Conforme ressaltado por Carvalho Filho et al. (2006), o óleo essencial de manjericão, possui alta concentração de linalol, valorizado no mercado internacional e amplamente utilizado nas indústrias de condimentos e cosméticos.

Segundo Alves et al. (2003), a ISO (International Standard Organization) define óleos essenciais como óleos voláteis obtidos na maioria dos casos por destilação por arraste com vapor d'água, formados por misturas complexas terpênicos lipofílicas geralmente líquidos apresentam em sua grande maioria, aroma agradável e intenso.

$O$ estresse hídrico, nutricional e salino durante o cultivo do manjericão, pode gerar uma maior quantidade e qualidade do óleo essencial (Amaral et al., 1999; Bernstein et al., 2010; Pravuschi et al., 2010; Ekren et al., 2012), diante disso, objetivou-se avaliar o efeito da salinidade da água de irrigação, no teor, rendimento e constituintes químicos do óleo essencial de Ocimum basilicum L.

\section{MATERIAL E MÉTODO}

O experimento foi conduzido em casa de vegetação, no período de janeiro a março de 2013, na área experimental do Núcleo de Engenharia de Água e Solo da Universidade Federal do Recôncavo da Bahia, localizado no município de Cruz das Almas - BA, à latitude de 22॰42' S, longitude 47॰38'
W e altitude média de $220 \mathrm{~m}$. O clima da região é classificado como úmido a sub-úmido, com umidade relativa e temperatura média anual de $80 \%$ e $24{ }^{\circ} \mathrm{C}$, respectivamente, e pluviosidade média anual de 1.143 mm (D'ANGIOLELLA et al., 1998).

O delineamento experimental utilizado foi o inteiramente casualizado, constando de 5 (cinco) tratamentos, com 4 (quatro) repetições. Foi utilizada água de chuva, por possuir baixa condutividade elétrica (CEa), aproximadamente igual a 0,016 dS $\mathrm{m}^{-1}$, correspondente ao tratamento T1 (testemunha), os demais tratamentos, T2, T3, T4 e T5, foram obtidos pela adição de $\mathrm{NaCl}$ comum (sem iodo) à água doce, até atingir os níveis de CEa desejados; 1,2 - T2; 2,3 - T3; 3,4 - T4 e 4,5 dS m -1 $^{-T}$ T, medidos por meio de um condutivímetro. Esses tratamentos correspondem a níveis de estresse por $\mathrm{NaCl}$ de 0,1 T1; 12,0 - T2; 23,0 - T3; 34,0 - T4 e 45,0 - T5 mM.

O material de solo foi originado de um Latossolo Amarelo coeso (EMBRAPA, 1997), coletado da camada $0-20 \mathrm{~cm}$ na UFRB. O solo foi seco ao ar, destorroado, homogeneizado e posteriormente retirado uma amostra para análise química (Tabela 1) conforme metodologias recomendadas pela EMBRAPA (1997). Na Figura 1, apresenta-se a curva de retenção de água no solo com os valores de ajuste da equação de Genuchten (1980).

O ajuste da curva de retenção seguiu o modelo de Genuchten (1980), conforme a equação 1:

$$
\theta(\Psi \mathrm{m}) \quad \theta_{\mathrm{r}}+\frac{\theta_{\mathrm{s}}-\theta_{\mathrm{r}}}{\left[1+\left(\alpha \cdot \Psi_{\mathrm{m}}\right)^{n}\right]^{\mathrm{m}}}
$$

em que:

$\theta\left(\Psi_{m}\right)$ - teor de água como função do potencial matricial $\Psi(\mathrm{m}), \mathrm{cm}^{3} \mathrm{~cm}^{-3}$;

$\theta_{\mathrm{r}}$ - teor de água residual do solo, $\mathrm{cm}^{3} \mathrm{~cm}^{-3}$;

$\theta_{\mathrm{S}}$ - teor de água do solo saturado, $\mathrm{cm}^{3} \mathrm{~cm}^{-3}$;

$\alpha, n$ - parâmetros empíricos de ajuste da equação, sendo que $m=1-(1 / n)$ (MUALEM, 1976).

As mudas de manjericão foram adquiridas de matrizes hidropônicas, propagadas por estaquia em células de espuma fenólica durante sete dias, e após o enraizamento foram encaminhadas para um berçário em sistema hidropônico permanecendo

TABELA 1. Características química do solo

\begin{tabular}{|c|c|c|c|c|c|c|c|c|c|}
\hline $\mathrm{pH}$ & M.O. & $\mathrm{P}$ & $\mathrm{K}$ & $\mathrm{Ca}+\mathrm{Mg}$ & $\mathrm{Al}$ & $\mathrm{Na}$ & $\mathrm{H}+\mathrm{Al}$ & SB & CTC \\
\hline & $\mathrm{g} \mathrm{Kg}^{-1}$ & $\mathrm{mg} \mathrm{dm}^{-3}$ & & --------- & ----- & $\mathrm{d}_{\mathrm{c}} \mathrm{dm}$ & - & ----- & \\
\hline 6,50 & 15,52 & 16,00 & 0,12 & 1,30 & 0,10 & 0,10 & 3,25 & 1,54 & 4,79 \\
\hline
\end{tabular}

Rev. Bras. PI. Med., Campinas, v.17, n.4, supl. I, p.807-813, 2015. 


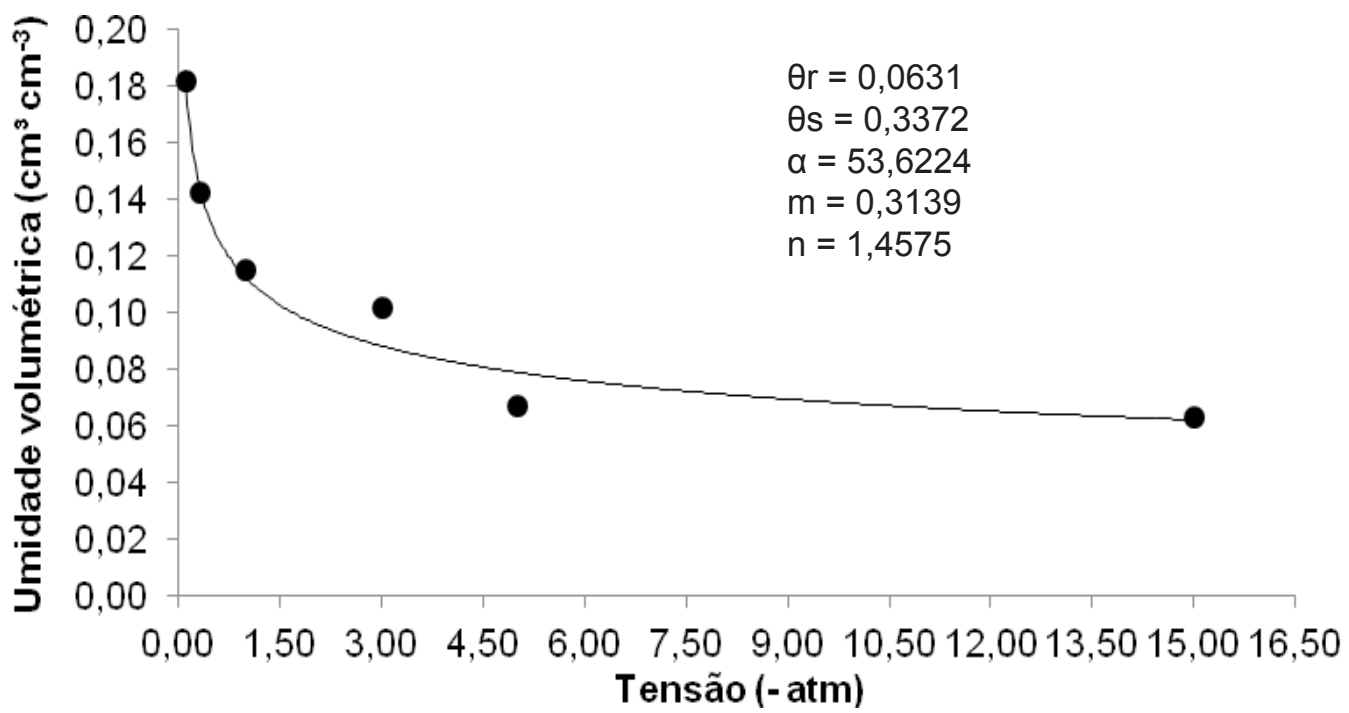

FIGURA 1. Curva de retenção de água no solo.

por um período de 20 dias, até atingir o momento de transplantio e transplantadas para recipientes, com volume de $0,1 \mathrm{~m}^{3}(100 \mathrm{I})$, preenchidos com solo, sendo cultivadas cinco plantas por recipiente. Os recipientes dispunham de um sistema de drenagem em sua base para permitir o fluxo do lixiviado. Os recipientes foram preenchidos com brita na parte inferior $(0,05 \mathrm{~m}$ do recipiente), recoberta com manta geotextil (bidim), com o objetivo de permitir o escoamento da água, impedindo a mistura do solo e da camada drenante. Por fim, acomodaramse seis camadas de solo, sempre adotando-se a mesma relação entre massa e volume de solo acomodado. Cada camada apresentava $5 \mathrm{~cm}$ de altura, totalizando $30 \mathrm{~cm}$ de altura e $108,00 \mathrm{~kg}$ de solo.

De acordo com as características químicas do solo, realizou-se uma adubação, utilizando húmus de minhoca (1 $\mathrm{kg}$ por recipiente), a partir da quarta camada do vaso $(15 \mathrm{~cm})$, pois segundo a literatura o sistema radicular do manjericão cresce em torno de 15 a $20 \mathrm{~cm}$, não necessitando adubar todo o solo do recipiente. A quantidade do adubo utilizado foi com base em recomendações para plantas medicinais segundo Trani et al., 2007. Optou-se pela utilização do húmus de minhoca, por esse se apresentar em forma coloidal e podendo influir em diversas propriedades físicas, químicas e biológicas do solo (Weinärtner et al., 2006), além de possuir vantagens como: Aumento da resistência das plantas a pragas e doenças, absorção favorecida dos nutrientes pelas raízes das plantas e entrada de água e ar facilitada. .

O manejo da irrigação foi realizado com base no monitoramento do potencial da água no solo medido por meio de tensiômetros, instalados a $20 \mathrm{~cm}$ de profundidade, em três recipientes de cada tratamento. Conectados com transdutores de pressão do tipo MPX 5100 (Motorola) para monitoramento continuado das variações de potencial da água no solo ao longo do tempo. Os dados de potencial da água no solo foram armazenados em um datalogger CR1000, Campbell Scientific.

Os valores de umidade foram obtidos a partir dos respectivos valores de potencial matricial, utilizando-se a curva de retenção de água no solo (Figura 1). A frequência de irrigação foi diária, aplicando-se ao solo o volume de água necessário para retornar a umidade do solo à capacidade de campo.

Aos 55 dias após o transplantio (DAT), as plantas foram colhidas, colocadas em sacos de papel e levadas a estufa de secagem com fluxo de ar forçado a $45^{\circ} \mathrm{C}$, por 10 dias até atingirem massas constantes. Após a secagem da parte aérea, foi realizada a pesagem da biomassa seca, misturada e moída em triturador elétrico de facas para extração do óleo essencial, realizada por hidrodestilação no Laboratório de Produtos Naturais (LAPRON) do Departamento de Ciências Exatas da Universidade Estadual de Feira de Santana - UEFS.

Resumidamente, o óleo essencial de $90 \mathrm{~g}$ de material vegetal seco e moído foram extraídos, com uma quantidade de água suficiente durante 2 horas num aparelho do tipo Clevenger. Os óleos extraídos foram secos com sulfato de sódio anidro, com objetivo de evitar perdas por hidrólise durante o armazenamento. O óleo foi recolhido, em seguida, armazenada em frascos selados a $-5{ }^{\circ} \mathrm{C}$ e protegido pela luz com folha de alumínio, até a realização da análise química, conforme Teles et al. $(2012,2013)$.

Primeiramente, separou-se $1 \mathrm{~g}$ do material 
seco e moído (o mesmo utilizado para extração do óleo), para a determinação do teor de umidade, em \%, que foi feita em triplicata (três leituras) no determinador de umidade (Série ID Versão 1.8 Marte ${ }^{\circledR}$.); neste aparelho as amostras vegetais foram secas a temperatura de $100^{\circ} \mathrm{C}$, até que não houvesse variação na pesagem de $0,1 \%$ em $30 \mathrm{~s}$.

$O$ teor do óleo essencial foi calculado (Equação 2) a partir da base livre de umidade (BLU), que corresponde ao volume $(\mathrm{mL})$ de óleo essencial em relação a massa seca.

$$
\mathrm{To}_{0}=\frac{\mathrm{Vo}}{\mathrm{Bm} \frac{(\mathrm{BmxU})}{100}} \times 100
$$

em que:

To - Teor de óleo (\%)

Vo - Volume de óleo extraído

$\mathrm{Bm}$ - Biomassa aérea vegetal

U - Umidade

O rendimento de óleo essencial foi obtido a partir da multiplicação entre o teor de óleo e a massa seca da parte aérea, conforme a Equação 3 a seguir:

$$
\text { Ro }=\text { ToxMSPA }
$$

em que:

Ro - rendimento de óleo essencial produzido

To - teor de óleo essencial

MSPA - massa seca da parte aérea da planta de manjericão, g por planta; (Santos et al., 2004)

Para a análise da composição química, 20 $\mathrm{mg}$ de óleo essencial foram diluídas em $1 \mathrm{~mL}$ de acetato de etila, e $1 \mu \mathrm{L}$ de volume foi injetado.

A determinação dos óleos essenciais foi analisada por cromatografia gasosa com detector de ionização de chama (CG/DIC). O Cromatógrafo utilizado foi um modelo Shimadzu® CG-2010 equipado com injetor automático AOC-20i, e um detector DIC. As análises foram realizadas com uma coluna capilar Rtx-5 (30 m x $0.25 \mathrm{~mm}$ ), espessura do filme $0.25 \mu \mathrm{m}$. As temperaturas do DIC e do injetor eram de 240 e $220^{\circ} \mathrm{C}$, respectivamente. $\mathrm{O}$ gás de arraste hélio, a uma taxa de fluxo de $1.2 \mathrm{~mL}$. $\mathrm{min}^{-1}$, com programa de temperatura do forno de $60{ }^{\circ} \mathrm{C}$ com um aumento de $3^{\circ} \mathrm{C} \cdot \mathrm{min}^{-1}$ até $240^{\circ} \mathrm{C}$, e mantida durante 20 min, split de 1:20.

As análises por cromatografia gasosa com detector de espectrometria de massa (CG/EM) foram realizadas em Cromatógrafo Shimadzu® CG2010 acoplado a um Espectrômetro de Massas CG/ MS-QP 2010 Shimadzu®, com injetor automático
AOC-20i, coluna capilar DB-5ms (30 m x $0.25 \mathrm{~mm}$ ), espessura do filme $0.25 \mu \mathrm{m}$, temperatura do injetor $220^{\circ} \mathrm{C}$, o gás de arraste hélio a uma taxa de fluxo de $1 \mathrm{~mL}$. min-1 ${ }^{-1}$ com temperatura da interface e da fonte de ionização de $240^{\circ} \mathrm{C}$, energia de ionização de 70 eV e a corrente de ionização de $0.7 \mathrm{kV}$, e programa de temperatura e split semelhante à descrita acima.

A identificação dos constituintes foi realizada por comparação dos índices de retenção (como índice de Kovats) de cada um dos picos e pelos dados de espectrometria de massas. Os índices foram calculados com a utilização de cromatogramas obtidos pela co-injeção da amostra com uma série homóloga de n-alcanos (C8 a C24). Cada pico do cromatograma foi também identificado pelo seu espectro de massas, pela comparação com a biblioteca do equipamento, pela consulta da literatura especializada (Adams, 2007; Joulain \& Konig, 1998) e pela injeção de padrões. Já a quantificação dos constituintes identificados foi obtida com base nas áreas dos picos cromatográficos correspondentes utilizando-se o método da normalização.

Para a análise dos resultados obtidos foi empregado o tratamento estatístico das características individuais. A análise de variância foi realizada pelo teste $F$ utilizando-se do teste de Tukey para a comparação entre médias. A análise da regressão polinomial foi utilizada para o desdobramento dos graus de liberdade dos teores de $\mathrm{NaCl}$.

\section{RESULTADO E DISCUSSÃO}

O aumento dos níveis de salinidade não alterou o teor de óleo essencial de manjericão, cuja variação foi de $0,87 \%$ a $1,00 \%$ e coeficiente de variação (CV) de 13,24\% (Tabela 2). Carrasco et al. (2007) cultivaram manjericão em sistema hidropônico NFT, com diferentes níveis de salinidade $(1,5 ; 3,0$ e 4,5 dS m-1) e não encontraram diferença significativa nos teores de óleo extraído das folhas frescas do manjericão, cuja variação foi de 0,24\% a $0,35 \%$. Pravuschi et al. (2010), não obtiveram diferenças nos teores de óleo extraído das folhas de manjericão $(0,71 \%$ a $2,18 \%)$ cultivado em solo sob condições de estresse hídrico.

O estresse salino não influenciou os teores de óleo essencial, provavelmente, devido ao fato da extração ter sido realizada em toda a parte aérea da planta. Comprovadamente, estudos indicam que as maiores concentrações de óleo essencial encontram-se nas folhas, cálices florais, canais secretores e pelos glandulares (Lawrence, 1992; Werker et al., 1993; Garlet, 2007; Bertolucci et al., 2008). Ainda de acordo com Andrade \& Casali (1999), o efeito sobre os produtos do metabolismo secundário parece variar bastante com o tipo, a 
TABELA 2. Média do teor do óleo essencial (\%), da parte aérea de plantas de Ocimum basilicum L., cultivadas com diferentes níveis de salinidade.

\begin{tabular}{ll}
\hline Tratamento $\left(\mathbf{d S ~}^{-1}\right)$ & Teor do óleo essencial (\%) \\
\hline T1 & 1,0099 a \\
T2 & 0,9949 a \\
T3 & 0,9798 a \\
T4 & 0,9346 a \\
T5 & 0,8743 a \\
\hline CV $(\%)$ & 13,24 \\
\hline
\end{tabular}

*Médias seguidas da mesma letra não diferem significativamente entre si a 5\% de probabilidade, pelo teste de Tukey.

intensidade e a duração do estresse, podendo aumentar ou diminuir o teor de óleos essenciais.

Conforme demonstrado na Figura 2, o rendimento de óleo essencial na parte aérea de Ocimum basilicum L., apresentou efeito significativo. Ocorreu uma redução de $12,93 \%$ a cada acréscimo unitário dos níveis de salinidade.

Trapp \& Croteau (2001), relatam que o rendimento do óleo pode variar de acordo com os fatores ambientais, material vegetal amostrado, ou método de extração. Ainda de acordo com Farias (1999), a localização geográfica, época da coleta, forma de cultivo, condições climáticas, idade do material vegetal, período e condições de armazenamento podem influenciar o rendimento e composição química de óleos essenciais por extratos de plantas.

A comparação das médias entre os tratamentos revelam que o rendimento do óleo da cultura é reduzido a partir de $1,2 \mathrm{dS} \mathrm{m}^{-1}$. As médias obtidas variaram de 0,23 a $0,54 \mathrm{~mL}$ por planta. Respostas similares foram encontradas por Carrasco et al. (2007), em cultivo de manjericão em sistema hidropônico NFT, com diferentes salinidades $\left(1,5 ; 3,0\right.$ e $4,5 d^{\prime}$ m $\left.^{-1}\right)$ e obtiveram variação entre 0,24 e $0,35 \mathrm{~mL}$ por planta com o aumento da salinidade.

Foram identificados 24 compostos químicos do óleo essencial, destes, os compostos E-Cinamato de metila $(55,4 \%)$, Metil chavicol $(12,63 \%)$, 1,8 -Cineol $(7,73 \%)$ e Z-Cinamato de metila $(3,9 \%)$ foram predominantes no óleo essencial de Ocimum basilicum L. cultivado sob condições de estresse salino. De acordo com Vieira et al. (2001) já foram encontrados os seguintes valores para espécie do gênero Ocimum encontrados no mercado e utilizados na medicina popular, linalol $(49,7 \%), 1,8$ cineol $(22 \%)$, metilchavicol $(47 \%)$ e cinamato de metila (65,5\%). Roque (1991) analisou a composição dos óleos essenciais de $O$. basilicum, e destacou que os compostos predominantes foram: linalol (52 a $60 \%$ ), eugenol (9 a 18\%), metil chavicol (2 a 4\%),

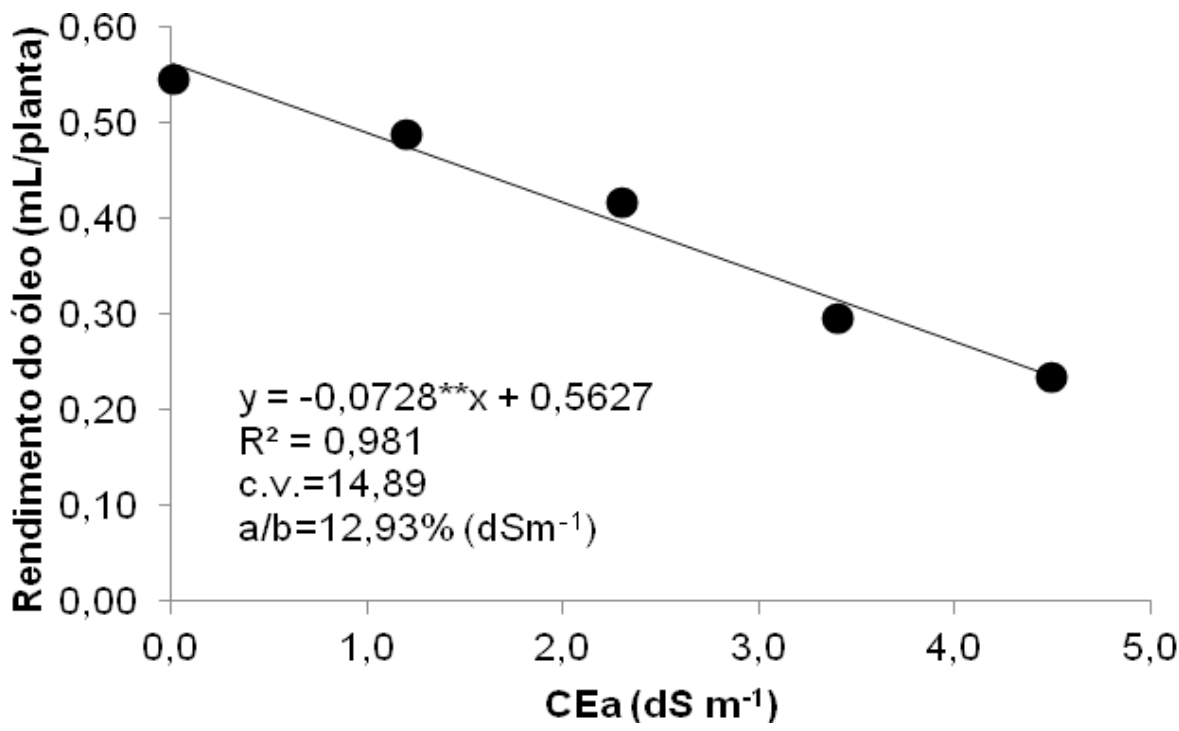

FIGURA 2. Rendimento do óleo essencial em função da salinidade de água de irrigação (CEa). "'Significativo pelo teste $\mathrm{F}$ a $1 \%$ de probabilidade; $\mathrm{R}^{2}$ = coeficiente de determinação; c.v. = coeficiente de variação; $\mathrm{a} / \mathrm{b}$ = redução ou incremento linear na variável y em função de cada aumento unitário na salinidade da água.

Rev. Bras. PI. Med., Campinas, v.17, n.4, supl. I, p.807-813, 2015. 
TABELA 3. Resultado da análise de variância aplicado aos compostos químicos predominantes no óleo essencial de manjericão.

\begin{tabular}{lll}
\hline Variável & C.V.(\%) & Pr>Fc \\
\hline 1,8 Cineol (\%) & 18,37 & $0,6832^{\text {ns }}$ \\
Metil Chavicol (\%) & 16,94 & $0,1395^{\text {ns }}$ \\
Z-Cinamato de Metila (\%) & 8,62 & $0,0327^{*}$ \\
E-Cinamato de Metila (\%) & 12,50 & $0,1267^{\text {ns }}$ \\
\hline
\end{tabular}

*Significativo pelo teste $\mathrm{F}$ a $5 \%$ de probabilidade; $\mathrm{ns}=$ não significativo; $\mathrm{c} . \mathrm{v} .=$ coeficiente de variação; $\mathrm{Pr}>\mathrm{Fc}=$ probabilidade correspondente a um $\mathrm{F}$ maior que $\mathrm{F}$ calculado

metil cinamato (2 a 5\%) e cineol (3 a $6 \%)$.

E-Cinamato de metila foi o composto encontrado em maior quantidade em todos os tratamentos, destacando-se como o composto majoritário.

As relações entre os compostos de maior predominância no óleo do manjericão e os níveis de salinidade não foram significativas, com exceção do composto Z-Cinamato de Metila, que apresentou significância, conforme demonstrado na Tabela 3, porém não se ajustou a nenhum modelo de regressão, provavelmente devido aos baixos coeficientes de determinação obtidos.

\section{CONCLUSÃO}

O aumento dos níveis de salinidade com adição de $\mathrm{NaCl}$, não alterou os teores de óleo essencial da parte aérea de Ocimum basilicum L.;

A irrigação com água de salinidade igual ou superior a $1,2 \mathrm{dS} \mathrm{m}^{-1}$ reduz o rendimento do óleo essencial das plantas de manjericão em comparação a irrigação realizada com água não salina;

Os compostos químicos do óleo essencial encontrados em maior quantidade na parte aérea do manjericão foram: E-Cinamato de metila $(55,4 \%)$, Metil chavicol (12,63\%), 1,8-Cineol $(7,73 \%)$ e Z-Cinamato de metila (3,9\%). Destes, o E-Cinamato de metila foi o principal composto constituinte.

\section{AGRADECIMENTO}

À Universidade Federal do Recôncavo da Bahia, à Universidade Estadual de Feira de Santana, à FAPESB e ao CNPq, pelo suporte financeiro.

\section{REFERÊNCIAS}

ADAMS, R.P. Identification of essential oil components by gas chromatography/mass spectrometry. 4.ed. Carol Stream, Illinois-USA: Allured Publishing Corporation, 2007. 804p.

ALVES, P.B. et al. Linalol de Ocimum basilicum (Manjericão) e características agronômicas de acessos do banco ativo de germoplasma da UFS. Cadernos UFS-Química, v.5, n.2, p.7-14, 2003.
AMARAL, J.F.T. et al. Deficiências de macronutrientes, Fe e B em manjericão, (Ocimum sp.) em cultivo hidropônico. Revista Ceres, v.46, n.265, p.297-308, 1999.

ANDRADE, F.M.C.; CASALI, V.W.D. Plantas medicinais e aromáticas: relação com ambiente, colheita e metabolismo secundário. (Ed.). Viçosa: Universidade Federal de Viçosa, Departamento de Fitotecnia, 1999. 139p.

AZEVEDO, C.D.; MOURA, M.A. Cultivos de plantas medicinais: guia prático. Niterói: Programa Rio Rural, 2010. 19p.

BERNSTEIN, N. et al. Salinity-induced changes in essential oil, pigments and salts accumulation in sweet basil (Ocimum basilicum) in relation to alterations of morphological development. Annals of Applied Biology, v.156, n.2, p.167-177, 2010.

BERTOLUCCI, S.K.V.; LAMEIRA, O.A.; PINTO, J.E.B.P. Guia das plantas medicinais. In. LAMEIRA, O. A.; PINTO, J. E. B. P. Plantas medicinais: do cultivo, manipulação e uso à recomendação popular. (Ed.). Belém, PA: Embrapa Amazônia Oriental, 2008, p.159-244.

CARRASCO, G. et al. Efecto de la conductividade elétrica de la solución nutritiva sobre el rendimento y contenido de aceite esencial en albahaca cultivada en NFT. Idesia, v.25, n.2, p.59-62, 2007.

CARVALHO FILHO, J.L.S. et al. Influence of the harvesting time, temperature and drying period on basil ( Ocimum basilicum L.) essential oil. Revista Brasileira de Farmacognosia, v.16, n.4, p.24-30, 2006.

D’ANGIOLELLA, G.L.B. et al. Tendências climáticas para os Tabuleiros Costeiros da região de Cruz das Almas, BA. In: CONGRESSO BRASILEIRO DE ENGENHARIA AGRÍCOLA, 27, 1998, Poços de Caldas. Anais. Lavras: SBEA, 1998. p.43-45.

EKREN, S. et al. The effect of different irrigation water levels on yield and quality characteristics of purple basil (Ocimum basilicum L.). Agricultural Water Management, v.109, p.155-161, 2012.

EMBRAPA. Manual de métodos de análise de solo. 2.ed. Rio de Janeiro: Centro Nacional de Pesquisa de Solo, 1997. 212p.

FARIAS, M.R. Em Farmacognosia da planta ao medicamento. In: SIMÕES, C.M.O.; SCHENKEL, E.P.; GOSMANN, G.; de MELLO, J.C.P.; MENTZ, L.A.; PETROVICK. Avaliação da Qualidade de matéria primas vegetais. 5.ed. Porto Alegre: UFSC e UFRGS, 1999.

GARLET, T.M.B. Produtividade, teor e composição do óleo essencial de espécies de Mentha L. (Lamiaceae) cultivadas em hidroponia com variação de potássio. 2007. 112p. Tese (Doutorado - Área de concentração

Rev. Bras. PI. Med., Campinas, v.17, n.4, supl. I, p.807-813, 2015. 
em Produção Vegetal) - Centro de Ciências Rurais, Universidade Federal de Santa Maria, Santa Maria.

GENUCHTEN, M.T. A closed form equation for predicting the hydraulic conductivity of unsaturated soils. Soil Science Society of America Journal, v.44, p.892898, 1980.

JOULAIN, D.; KÖNIG, W.A. The atlas of spectral data of sesquiterpene hydrocarbons. HamburgGermany: EB-Verlag, 1998. 658p.

LAWRENCE, B.M. Chemical components of Labiatae oils and their exploitation. In: HARLEY, R.M.; REINOLDS, T. Advances in Labiatae science. (Eds.). Kew, UK: Royal Botanical Gardens, 1992, p.399-436.

LORENZI, H; MATOS, F.J.A. Plantas Medicinais no Brasil: nativas e exóticas. 2.ed. Nova Odessa, São Paulo: Instituto Plantarum, 2008. 544p.

MUALEM, Y. A new model for predicting the hydraulic conductivity of unsaturated porous media. Water Resources Research, v.12, n.3, p.513-522, 1976.

PRAVUSCHI, P.R. et al. Efeito de diferentes lâminas de irrigação na produção de óleo essencial do manjericão (Ocimum basilicum L.). Acta Scientiarum. v.32, n.4, p.687-693, 2010.

ROQUE, O.L.R. Composição do óleo essencial de Ocimum basilicum L. cultivado. Boletim da Faculdade de Farmácia de Coimbra, v.15, n.1, p.47-51, 1991.

RHOADES, J.D.; KANDIAH, A.; MASHAL, A.M. Uso de águas salinas para produção agrícola. Campina Grande: UFPB, 2000. 117p.

SANTOS, A.S. et al. Descrição de sistema e de métodos de extração de óleos essenciais e determinação de umidade de biomassa em laboratório. Belém: Embrapa, 2004. 6p. (Comunicado Técnico, 99).

SILVA, J.V. et al. Physiological responses of $\mathrm{NaCl}$ stressed cowpea plants grown in nutrient solution supplemented with $\mathrm{CaCl}_{2}$. Brazilian Journal of Plant Physiology, v.15, n.2, p.99-105, 2003.

TELES, S. et al. Geographical origin and drying methodology may affect the essential oil of Lippia alba (Mill) N.E. Brown. Industrial Crops and Products, 37, 247-252, 2012.

TELES, S. et al. Effect of geographical origin on the essential oil content and composition of fresh and dried Mentha $\times$ villosa Hudson leaves. Industrial Crops and Products, 46, 1-7, 2013.

TRANI, P.E. et al. Hortaliça e Plantas medicinais: manual prático. Campinas: Instituto Agronômico, 2007. 72p. (Boletim Técnico IAC, 199).

TRAPP, S.A.; CROTREAU, R.D. Genomic organization of plant terpene synthases and molecular evolutionary implications. Genetic. v.158, p.811-32, 2001.

VIEIRA, R.F. et al. Genetic diversity of Ocimum grantissimum L. based on volatile oil constituents, flavonoids and RAPD markers. Biochemical Systematic Ecologic., v.29, p.287-304, 2001.

WEINÄRTNER, M.A.; ALDRIGHI, C.F.S.; MEDEIROS, C.A.B. Práticas Agroecológicas: Adubação orgânica. Pelotas, 2006. 20p.

WERKER, E. et al. Glandular hairs and essential oil in developing leaves of Ocimum basilicum L. (Lamiaceae). Annals of Botany, v.71, p.43-50, 1993. 